\title{
Effect of biaxial strain on the electrical and magnetic properties of (001)
}

\section{$\mathrm{La}_{0.7} \mathrm{Sr}_{0.3} \mathrm{MnO}_{3}$ thin films}

C. Adamo, X. Ke, H. Q. Wang, H. L. Xin, T. Heeg, M. E. Hawley, W. Zander, J. Schubert, P. Schiffer, D. A. Muller, L. Maritato, and D. G. Schlom

Citation: Appl. Phys. Lett. 95, 112504 (2009);

View online: https://doi.org/10.1063/1.3213346

View Table of Contents: http://aip.scitation.org/toc/apl/95/11

Published by the American Institute of Physics

\section{Articles you may be interested in}

Strain-dependent magnetic phase diagram of epitaxial $\mathrm{La}_{0.67} \mathrm{Sr}_{0.33} \mathrm{MnO}_{3}$ thin films

Applied Physics Letters 76, 2421 (2000); 10.1063/1.126363

Quantifying strain dependence in "colossal" magnetoresistance manganites

Journal of Applied Physics 83, 1588 (1998); 10.1063/1.367310

The role of strain in magnetic anisotropy of manganite thin films

Applied Physics Letters 71, 140 (1998); 10.1063/1.119454

Thickness-dependent magnetotransport in ultrathin manganite films

Applied Physics Letters 74, 3017 (1999); 10.1063/1.124050

Nearly total spin polarization in $\mathrm{La}_{2 / 3} \mathrm{Sr}_{1 / 3} \mathrm{MnO}_{3}$ from tunneling experiments

Applied Physics Letters 82, 233 (2003); 10.1063/1.1534619

Strain-induced magnetic stripe domains in $\mathrm{La}_{0.7} \mathrm{Sr}_{0.3} \mathrm{MnO}_{3}$ thin films

Applied Physics Letters 82, 1434 (2003); 10.1063/1.1556967

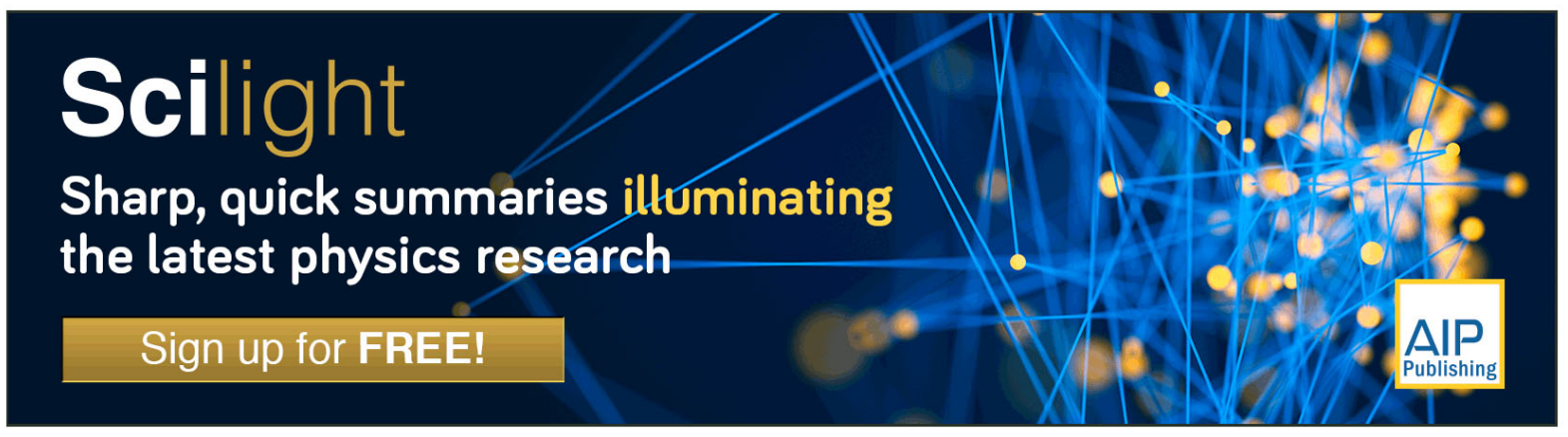




\title{
Effect of biaxial strain on the electrical and magnetic properties of (001) $\mathrm{La}_{0.7} \mathrm{Sr}_{0.3} \mathrm{MnO}_{3}$ thin films
}

\author{
C. Adamo, ${ }^{1}$ X. Ke, ${ }^{2}$ H. Q. Wang, ${ }^{3}$ H. L. Xin, ${ }^{3}$ T. Heeg, ${ }^{1}$ M. E. Hawley, ${ }^{4}$ W. Zander, ${ }^{5}$ \\ J. Schubert, ${ }^{5}$ P. Schiffer, ${ }^{2}$ D. A. Muller, ${ }^{3}$ L. Maritato, ${ }^{6}$ and D. G. Schlom ${ }^{1, a}$ \\ ${ }_{1}^{1}$ Department of Materials Science and Engineering, Cornell University, Ithaca, New York 14853-1501, USA \\ ${ }^{2}$ Department of Physics and Materials Research Institute, The Pennsylvania State University, \\ University Park, Pennsylvania 16802, USA \\ ${ }^{3}$ School of Applied and Engineering Physics, Cornell University, Ithaca, New York 14853, USA \\ ${ }^{4}$ Materials Science and Technology Division (MST-8), Los Alamos National Laboratory, Los Alamos, \\ New Mexico 87545, USA \\ ${ }^{5}$ Institute of Bio-and Nano-Systems (IBN1-IT) and JARA-Fundamentals of Future Information Technology, \\ Research Centre Jülich, Jülich D-52425, Germany \\ ${ }^{6}$ Dipartimento di Matematica ed Informatica, Università di Salerno and CNR-INFM Salerno, \\ 84081 Baronissi (SA), Italy
}

(Received 25 March 2009; accepted 7 August 2009; published online 17 September 2009)

\begin{abstract}
We have studied the effect of biaxial strain on thin films of (001) $\mathrm{La}_{0.7} \mathrm{Sr}_{0.3} \mathrm{MnO}_{3}$. We deposited films by reactive molecular-beam epitaxy on different single crystalline substrates, varying the substrate-induced biaxial strain from $-2.3 \%$ to $+3.2 \%$. Magnetization and electrical transport measurements reveal that the dependence of the Curie temperature on biaxial strain is in very good agreement with the theoretical predictions of Millis et al. [J. Appl. Phys. 83, 1588 (1998)]. @ 2009 American Institute of Physics. [doi:10.1063/1.3213346]
\end{abstract}

In bulk form or unstrained films, the compound $\mathrm{La}_{0.7} \mathrm{Sr}_{0.3} \mathrm{MnO}_{3}$ exhibits colossal magnetoresistance $(\mathrm{CMR})^{1-4}$ well above room temperature. In epitaxial films, the different lattice spacing of an underlying substrate can be used to impose a biaxial strain state, which influences the Jahn-Teller effect in $\mathrm{La}_{0.7} \mathrm{Sr}_{0.3} \mathrm{MnO}_{3}$.

Millis et al. ${ }^{5}$ proposed an analytical model to describe the effects of biaxial strain $\left(\varepsilon_{x x}\right.$ and $\left.\varepsilon_{y y}\right)$ on the magnetotransport properties of CMR manganites. In this model, the Curie temperature $\left(T_{C}\right)$ depends on two parameters: (1) the bulk compression $\varepsilon_{B}=\frac{1}{3}\left(2 \varepsilon_{x x}+\varepsilon_{z z}\right)$ (assuming $\left.\varepsilon_{x x}=\varepsilon_{y y}\right)$, and (2) the biaxial distortion $\varepsilon^{*}=1 / 2\left(\varepsilon_{z z}-\varepsilon_{x x}\right)$, where $\varepsilon_{x x}=\left(a_{x x}\right.$ $\left.-a_{\text {bulk }}\right) / a_{\text {bulk }}$ and $\varepsilon_{z z}=\left(a_{z z}-a_{\text {bulk }}\right) / a_{\text {bulk }}$ are the pseudocubic in-plane and out-of-plane strain, respectively.

Several studies have investigated the effects of substrateinduced biaxial strain on the magnetotransport properties of manganite thin films. ${ }^{6-16}$ In this work, we investigate the magnetotransport properties of fully commensurate epitaxial $\mathrm{La}_{0.7} \mathrm{Sr}_{0.3} \mathrm{MnO}_{3}$ thin films grown by reactive molecular-beam epitaxy. Our films span a biaxial strain range from $-2.3 \%$ to $+3.2 \%$, and we find excellent agreement between the measured Curie temperatures and the model of Millis et al. ${ }^{5}$ The films were grown on nine commercially available perovskite substrates: (100) ${ }_{p} \mathrm{LaAlO}_{3},(001) \mathrm{LaSrGaO}_{4}$, (110) $\mathrm{NdGaO}_{3}$, (100) $\left(\mathrm{LaAlO}_{3}\right)_{0.3}-\left(\mathrm{SrAl}_{0.5} \mathrm{Ta}_{0.5} \mathrm{O}_{3}\right)_{0.7}$ (LSAT), (100) $\mathrm{SrTiO}_{3}$, (110) $\mathrm{DyScO}_{3},(110) \mathrm{GdScO}_{3},(110) \mathrm{SmScO}_{3}$, and (110) $\mathrm{NdScO}_{3}$, where the subscript $p$ indicates pseudocubic indices. The homogenously strained commensurate epitaxial thin films were all $22 \mathrm{~nm}$ thick, except for the film on $\mathrm{NdScO}_{3}$. As (110) $\mathrm{NdScO}_{3}$ is the most mismatched (+3.2\%) substrate used in this study, the $\mathrm{La}_{0.7} \mathrm{Sr}_{0.3} \mathrm{MnO}_{3}$ had to be thinner (10 $\mathrm{nm}$ ) to achieve a commensurately strained film.

The $\mathrm{La}_{0.7} \mathrm{Sr}_{0.3} \mathrm{MnO}_{3}$ films were grown by codepositing the constituent elements including purified ozone (at a back-

${ }^{a)}$ Electronic mail: schlom@cornell.edu. ground partial pressure of $5 \times 10^{-7}$ Torr) at a substrate temperature of $700{ }^{\circ} \mathrm{C}$, measured by an optical pyrometer. Reflection high-energy electron diffraction (RHEED) oscillations were used to confirm the overall growth rate. ${ }^{17}$ RHEED patterns observed during growth were consistent with flat surfaces. This was confirmed by atomic force microscopy measurements revealing a root mean square roughness of the films ranging from $0.21 \mathrm{~nm}$ on (110) $\mathrm{DyScO}_{3}$ to $0.54 \mathrm{~nm}$ on $(100)_{p} \mathrm{LaAlO}_{3}$.

$\mathrm{X}$-ray diffraction (XRD) was used to determine the inplane and out-of-plane lattice parameters using asymmetric and symmetric film reflections. Cross-sectional annular dark field (ADF) scanning transmission electron microscopy (STEM) images were recorded on a $200 \mathrm{kV}$ FEI Tecnai F20-ST STEM with a minimum probe size of 1.6-1.9 $\AA$ and the chemical composition across the interface was probed at the atomic scale using spatially resolved electron energy loss spectroscopy. The transport properties were measured using a standard four-probe van der Pauw geometry, and the magnetic measurements were performed by means of superconducting quantum interference device magnetometry.

Figure 1 shows the $\theta-2 \theta$ XRD scans of the (001)oriented $\mathrm{La}_{0.7} \mathrm{Sr}_{0.3} \mathrm{MnO}_{3}$ thin films. The arrows indicate the 002 film peaks, which shift from the bulk unstrained value of $\mathrm{La}_{0.7} \mathrm{Sr}_{0.3} \mathrm{MnO}_{3}$ in response to the in-plane spacings of the substrates upon which the films are grown. In addition to the intense substrate peaks and 002 film peaks, thickness (Kiesel) fringes also are apparent. In all of the samples, the $2 \theta$ position of the asymmetric XRD peaks in combination with the $2 \theta$ position of the out-of-plane $00 \ell$ peaks indicate that the in-plane lattice parameters of the films equal those of the substrates, i.e., the films are all commensurate.

The films also show rocking curve widths in $\omega$ limited by the quality of the substrates on which they are grown. As an example, the full width at half maximum of the rocking curve of the 002 reflection of the $\mathrm{La}_{0.7} \mathrm{Sr}_{0.3} \mathrm{MnO}_{3}$ film on $\mathrm{SrTiO}_{3}$ is $0.011^{\circ}$; on $\mathrm{NdGaO}_{3}$ it is $0.005^{\circ}$ and on $\mathrm{DyScO}_{3}$ it 


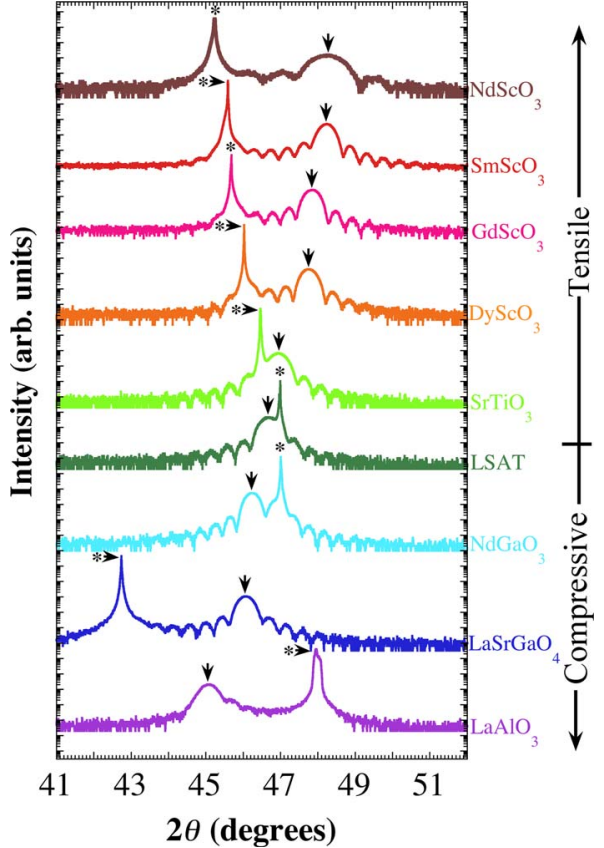

FIG. 1. (Color online) $\theta-2 \theta$ XRD scans of $22 \mathrm{~nm}$ thick $\mathrm{La}_{0.7} \mathrm{Sr}_{0.3} \mathrm{MnO}_{3}$ films grown on different substrates $\left(10 \mathrm{~nm}\right.$ thick on $\left.\mathrm{NdScO}_{3}\right)$. These substrates impose strains $\varepsilon$ ranging from $-2.3 \%\left(\mathrm{LaAlO}_{3}\right)$ to $+3.2 \%\left(\mathrm{NdScO}_{3}\right)$. The arrows $(\downarrow)$ indicate the $002 \mathrm{La}_{0.7} \mathrm{Sr}_{0.3} \mathrm{MnO}_{3}$ film peaks.

is $0.003^{\circ}$. These are the narrowest rocking curve widths ever reported for $(\mathrm{La}, \mathrm{Sr}) \mathrm{MnO}_{3}$ films or single crystals. ${ }^{12}$

Figure 2(a) shows an ADF-STEM image of a $\mathrm{La}_{0.7} \mathrm{Sr}_{0.3} \mathrm{MnO}_{3}$ thin film grown on $\mathrm{DyScO}_{3}$. It further confirms the coherent nature of the interface. Some interdiffusion between the $\mathrm{La}_{0.7} \mathrm{Sr}_{0.3} \mathrm{MnO}_{3}$ film and the $\mathrm{DyScO}_{3}$ substrate was observed [Fig. 2(b)].

The ratio $a_{z z} / a_{x x}$ between the measured out-of-plane and in-plane film lattice parameters as a function of the substrate lattice parameter is shown in Fig. 3(a). The regular behavior of the tetragonal distortion, observed in Fig. 3(a), is a clear indication of the dominant role played by biaxial strain on the structural lattice deformations of the films. This is further confirmed by the results shown in Fig. 3(b), where Poisson's ratio of the deposited $\mathrm{La}_{0.7} \mathrm{Sr}_{0.3} \mathrm{MnO}_{3}$ films is determined by

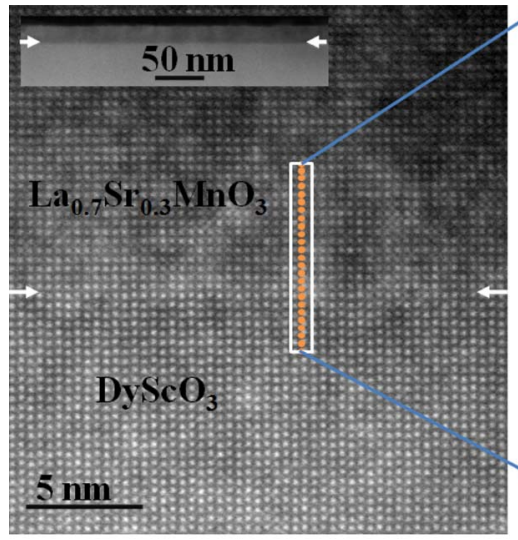

(a)

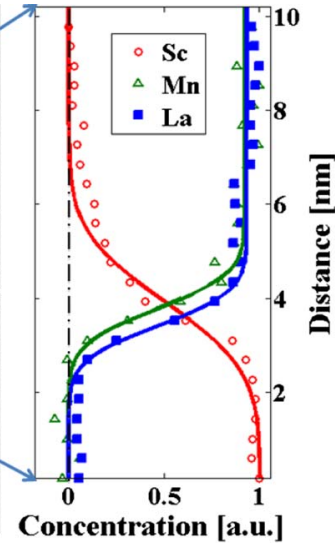

(b)
FIG. 2. (Color online) (a) ADF-STEM image of a $\mathrm{La}_{0.7} \mathrm{Sr}_{0.3} \mathrm{MnO}_{3}$ film deposited on (110) $\mathrm{DyScO}_{3}$, viewed along the [001] zone axis of the substrate $\left(\varepsilon_{x x}=+1.6 \%\right)$. The inset STEM image shows the film across a $300 \mathrm{~nm}$ field of view. (b) Concentration profiles of $\mathrm{Sc}, \mathrm{Mn}$, and $\mathrm{La}$ across the $\mathrm{La}_{0.7} \mathrm{Sr}_{0.3} \mathrm{MnO}_{3} / \mathrm{DyScO}_{3}$ interface (arrowed in STEM images).

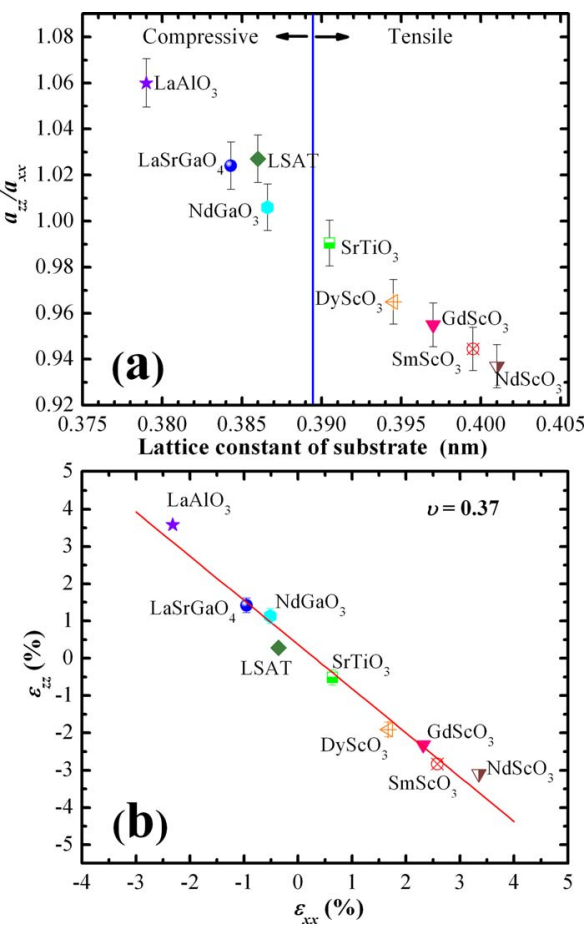

FIG. 3. (Color online) (a) Ratio between the out-of-plane $\left(a_{z z}\right)$ and in-plane $\left(a_{z z}\right)$ lattice spacing of commensurately $\mathrm{La}_{0.7} \mathrm{Sr}_{0.3} \mathrm{MnO}_{3}$ films as a function of the pseudocubic in-plane substrate lattice parameter. (b) The out-of-plane lattice strain $\varepsilon_{z z}$ as a function of the in-plane lattice strain $\varepsilon_{x x}$.

plotting the out-of-plane lattice strain versus the in-plane lattice strain. The linear dependence of the data points in Fig. 3(b) rules out any major role of interfacial effects (chemical interdiffusion, charge transfer) on the structural lattice deformation of the films and illustrates the dominant influence of the substrate-induced strain. Poisson's ratio can be calculated from $\nu=1 /\left(1-2 \varepsilon_{x x} / \varepsilon_{z z}\right){ }^{18} \varepsilon_{x x} / \varepsilon_{z z}$ is estimated by a linear fit (red line) to the data in Fig. 3(b) from which the value $\nu=0.37 \pm 0.01$ is obtained. This is the largest range of substrate induced strain over which Poisson's ratio has ever been calculated for a CMR manganite. The obtained value is in reasonable agreement with the typical $\nu$ value for manganites. ${ }^{7,19}$

The influence of biaxial strain on the temperature dependence of the resistivity $\rho(T)$ of the thin films grown on various substrates is shown in Fig. 4(a). The data clearly indicate that strain has a significant effect on the metal-insulator (MI) transition temperature. In the case of thin films with a small amount $\left(\left|\varepsilon_{x x}\right| \leq 0.6 \%\right)$ of strain $\left(\mathrm{SrTiO}_{3}\right.$, LSAT, and $\mathrm{NdGaO}_{3}$ ), the low temperature resistivity values $\rho$ $<0.1 \mathrm{~m} \Omega \mathrm{cm}$ are comparable to those measured on single crystals, confirming the high quality of these samples. ${ }^{20}$ The MI transition temperature $T_{\mathrm{MI}}$ is higher than $390 \mathrm{~K}$ for the samples grown on $\mathrm{NdGaO}_{3}\left(\varepsilon_{x x}=-0.5 \%\right)$ and LSAT $\left(\varepsilon_{x x}=-0.4 \%\right)$. The film grown on $\mathrm{SrTiO}_{3}\left(\varepsilon_{x x}=+0.6=\%\right)$ shows a $T_{\mathrm{MI}}$ around $370 \pm 10 \mathrm{~K}$. $T_{\mathrm{MI}}$ is reduced as the magnitude of the substrate mismatch increases, as in the case of the film on $\mathrm{DyScO}_{3}\left(\varepsilon_{x x}=+1.6 \%\right)$. The film deposited on $\mathrm{LaAlO}_{3}\left(\varepsilon_{x x}=-2.3 \%\right)$ shows an insulating behavior over the entire temperature range, in agreement with previous work. ${ }^{6,17}$ The films grown on $\mathrm{GdScO}_{3} \quad\left(\varepsilon_{x x}=+2.3 \%\right)$, $\mathrm{SmScO}_{3} \quad\left(\varepsilon_{x x}=+2.7 \%\right)$, and $\mathrm{NdScO}_{3} \quad\left(\varepsilon_{x x}=+3.2 \%\right)$ show room temperature resistivity values of $\approx 1 \Omega \mathrm{cm}$. Due to their high resistivities these $\rho(T)$ curves have been measured 

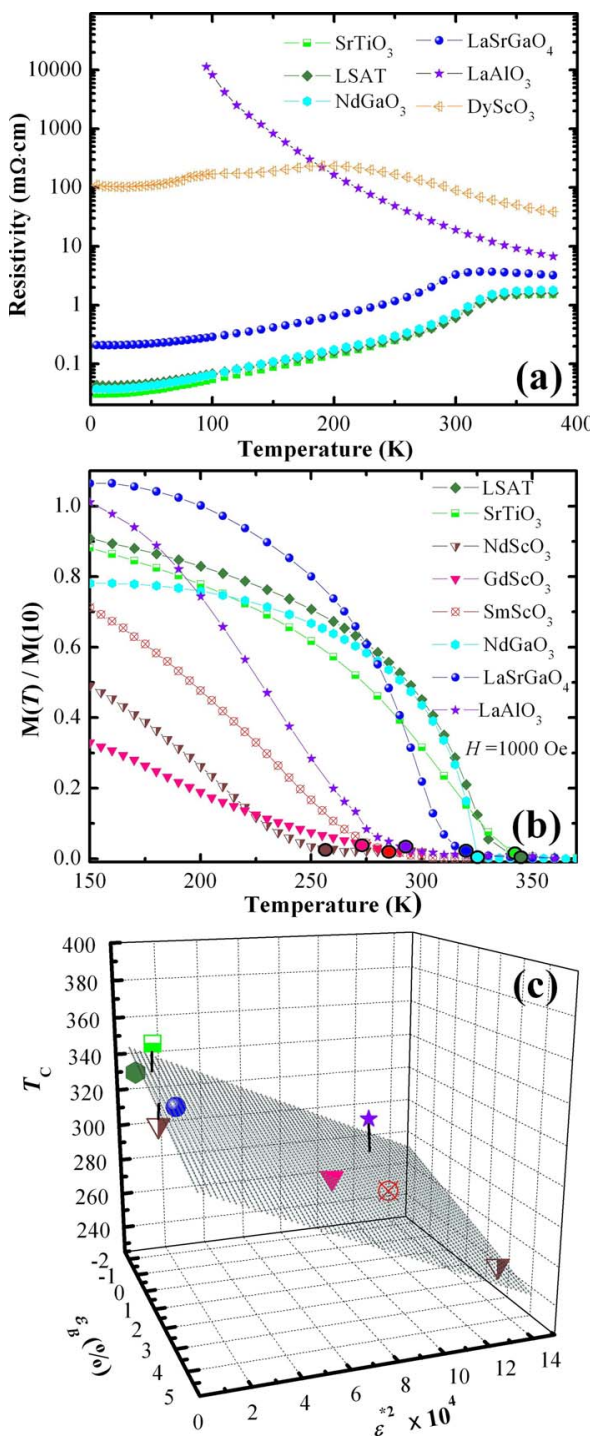

FIG. 4. (Color online) (a) Resistivity vs temperature behavior, at zero applied magnetic field, of $22 \mathrm{~nm}$ thick $\mathrm{La}_{0.7} \mathrm{Sr}_{0.3} \mathrm{MnO}_{3}$ films on different substrates $\left(10 \mathrm{~nm}\right.$ thick on $\mathrm{NdScO}_{3}$ ). (b) The temperature dependence of the magnetization normalized at $10 \mathrm{~K}$ of samples cooled in a 1000 Oe field of $H=1000$ Oe. Circles indicate the Curie temperature, $T_{C}$. (c) The Curie temperature $T_{C}$ vs the $\varepsilon_{B}$ and $\varepsilon^{*}$ strains. The best fit plane to the data is also shown.

over a reduced range of temperatures and are not reported in Fig. 4(a). In Fig. 4(b), we plot the magnetization, after subtracting the magnetization of the bare substrates. The samples were cooled down to $10 \mathrm{~K}$ in an applied field of 1000 Oe. The data were taken during warming up. For the films grown on the rare earth scandate substrates no magnetic field was applied due to the large paramagnetic background signal. For the other samples 1000 Oe was applied also during warming. The circles indicate the Curie temperature, defined as the temperature where the $M(T)$ of the film first deviates from $M(T)$ of the bare substrate.

In the model proposed by Millis et al. ${ }^{5}$ the effects of strain on the Curie temperature are described by the formula

$$
T_{c}\left(\varepsilon_{B}, \varepsilon^{*}\right)=T_{c}(0,0)\left[1-\alpha \varepsilon_{B}-b \varepsilon^{* 2}\right],
$$

with $\alpha=\left(1 / T_{C}\right)\left[\left(d T_{C}\right) /\left(d \varepsilon_{B}\right)\right]$ and $b=\left(1 / T_{C}\right)\left[\left(d^{2} T_{C}\right) /\left(d \varepsilon^{* 2}\right)\right]$. The parameter $\varepsilon_{B}$ is associated with a uniform compressive (tensile) strain and tends to increase (decrease) the electron hopping probability, reducing the effect of the electron- lattice coupling. Therefore, depending on the sign of the strain the $T_{C}$ change associated with $\varepsilon_{B}$ will be positive or negative, respectively. The other source of strain, related to the biaxial distortion $\varepsilon^{*}$, will increase the Jahn-Teller splitting of the $e_{g}$ electron levels and will cause only a decrease in $T_{C}$. Typical values for $\alpha$ and $b$ in manganites were predicted to be around 6 and $1.4 \times 10^{3}$, respectively. ${ }^{5}$

In the case of $\mathrm{La}_{0.7} \mathrm{Sr}_{0.3} \mathrm{MnO}_{3}$ films, considerable disagreement exists in the literature on the values of $\alpha$ and $b$. Tsui et al. ${ }^{10}$ grew 25 and $50 \mathrm{~nm}$ thick $\mathrm{La}_{0.7} \mathrm{Sr}_{0.3} \mathrm{MnO}_{3}$ films on $\mathrm{LaAlO}_{3}, \mathrm{NdGaO}_{3}$, LSAT, and $\mathrm{SrTiO}_{3}$ substrates and found $\alpha$ and $b$ values around 2 and 70, respectively. In contrast, Ranno et al. ${ }^{14}$ found $b$ values around 2200 for 30, 60, and $80 \mathrm{~nm}$ thick $\mathrm{La}_{07} \mathrm{Sr}_{0.3} \mathrm{MnO}_{3}$ films grown on $\mathrm{SrTiO}_{3}$, while Angeloni et al. ${ }^{15}$ reported $b$ values around $10^{3}$ for 16 nm thick $\mathrm{La}_{0.7} \mathrm{Sr}_{0.3} \mathrm{MnO}_{3}$ films grown on $\mathrm{LaAlO}_{3}$. All of these data were taken on samples with different thicknesses (i.e., different and often inhomogeneous strain conditions due to progressive strain relaxation) and used a smaller number of substrate materials in comparison with the present study. In Fig. 4(b), a three-dimensional plot showing the $T_{C}$ behavior versus $\varepsilon_{B}$ and $\varepsilon^{*}$ of our commensurately strained $\mathrm{La}_{0.7} \mathrm{Sr}_{0.3} \mathrm{MnO}_{3}$ films is shown. The plane in the figure is obtained by fitting the experimental data using Eq. (1). From the fitting procedure we obtain $T_{C}(0,0)=345 \pm 9 \mathrm{~K}$, $\alpha=1.55 \pm 0.01$, and $b=1460 \pm 30$, in very good agreement with the predictions of Millis et al. ${ }^{5}$

We gratefully acknowledge the financial support from the National Science Foundation through the MRSEC program (Grant Nos. DMR-0520404 and DMR-0820404) and Grant No. DMR-0701582.

${ }^{1}$ R. Von Helmolt, J. Wecker, B. Holzapfel, L. Schultz, and K. Samwer, Phys. Rev. Lett. 71, 2331 (1993).

${ }^{2}$ S. Jin, T. H. Tiefel, M. McCormack, R. A. Fastnacht, R. Ramesh, and L. H. Chen, Science 264, 413 (1994).

${ }^{3}$ J. M. D. Coey, M. Viret, and S. Von Molnar, Adv. Phys. 48, 167 (1999).

${ }^{4}$ B. R. K. Nanda and S. Sathpathy, Phys. Rev. B 78, 054427 (2008).

${ }^{5}$ A. J. Millis, T. Darling, and A. Migliori, J. Appl. Phys. 83, 1588 (1998).

${ }^{6}$ S. J. Hibble, S. P. Cooper, A. C. Hannon, I. D. Fawcett, and M. Greenblatt, J. Phys.: Condens. Matter 11, 9221 (1999).

${ }^{7}$ Y. Lu, J. Klein, F. Herbstritt, J. B. Philipp, A. Marx, and R. Gross, Phys. Rev. B 73, 184406 (2006).

${ }^{8}$ T. Dhakal, J. Tosado, and A. Biswas, Phys. Rev. B 75, 092404 (2007).

${ }^{9}$ D. Rubi, S. Duhalde, M. C. Terzzoli, G. Leyva, G. Polla, P. Levy, F. Parisi, and R. R. Urbano, Physica B 320, 86 (2002).

${ }^{10}$ F. Tsui, C. Smoak, T. K. Nath, and C. B. Eom, Appl. Phys. Lett. 76, 2421 (2000).

${ }^{11}$ E. S. Vlakhov, K. A. Nenkov, T. G. Donchev, E. S. Mateev, and R. A. Chakalov, Vacuum 76, 249 (2004).

${ }^{12}$ A. Y. Petrov, C. Aruta, S. Mercone, C. Adamo, I. Alessandri, and L. Maritato, Eur. Phys. J. B 40, 11 (2004).

${ }^{13}$ P. Murugavel, J. H. Lee, J.-G. Yoon, T. W. Noh, J. S. Chung, M. Heu, and S. Yoon, Appl. Phys. Lett. 82, 1908 (2003).

${ }^{14}$ L. Ranno, A. Llobet, R. Tiron, and E. Favre-Nicolin, Appl. Surf. Sci. 188 170 (2002).

${ }^{15}$ M. Angeloni, G. Balestrino, N. G. Boggio, P. G. Medaglia, P. Orgiani, and A. Tebano, J. Appl. Phys. 96, 6387 (2004).

${ }^{16}$ J. Cibert, J. Bobo, and U. Lüders, C. R. Phys. 6, 977 (2005).

${ }^{17}$ J. O'Donnell, A. E. Andrus, S. Oh, E. V. Colla, and J. N. Eckstein, Appl. Phys. Lett. 76, 1914 (2000).

${ }^{18}$ J. F. Nye, Physical Properties of Crystals (Oxford University Press, New York, 1985), p. 143.

${ }^{19}$ G. Fehringer, S. Janes, M. Wildersohn, and R. Clasen, J. Eur. Ceram. Soc. 24, 705 (2004).

${ }^{20}$ R. Shiozaki, K. Takenaka, Y. Sawaki, and S. Sugai, Phys. Rev. B 63 , 184419 (2001). 